\title{
Concept for Mass Production of Complex Shaped Prototypes
}

\author{
Katarina Monkova ${ }^{1}$, Peter Monka ${ }^{2}$ \\ ${ }^{1}$ FMT TU with a seat in Presov, Department of technological devices design, Sturova 31, 08001 Presov, Slovakia \\ ${ }^{2}$ FMT TU with a seat in Presov, Department of manufacturing technologies, Sturova 31, 08001 Presov, Slovakia \\ katarina.monkova@tuke.sk
}

\begin{abstract}
In the article is described one of the concepts how to mass produce the complex-shaped part prepared as a prototype in manual way without the analytical and dimensional characteristics. The technology selection for production of the parts listed above in specific plant conditions is influenced by several requests as are for example material and shape of the part, number of parts, types of available machines, etc. The base for problem solution can be the computer aid, NC machines and modern equipments that are used within the method Reverse engineering. It is the process of obtaining a geometric CAD model from 3D points acquired by scanning existing object. Presented concept of data digitizing enables the automation of the production, so it can considerably decrease the economic cost and greatly affect the efficiency of the part manufacturing.

Index Terms - Prototype, Reverse Engineering, 3D canner, Digitizing, NC program.
\end{abstract}

\section{Introduction}

The only way for companies how to survive and prosper is their ability to achieve a good indicator of profit and their quickly respond to the market demands. In the mechanical engineering industry and manufacturing technologies, it holds much more. Being faster to market while increasing quality, this is a crucial competitive advantage of successful business future, which raises the need to address complex problems in all phases of development and production of selected products with the using of available technical, information and automation systems.

Significant utilization of new approaches in connection with technical progress permanently increases the pressure for the manufacturers to develop and make the products as soon as possible at the minimal cost and in required quality. The products have to be competitive, they have to be liable for qualitative and functional standard, efficacious design, and they must have regard for safety, ergonomic and other aspects, which decided about its marketability.

The substantial influence on the technical, social and economical level of manufacturing process has the computer technique implementation into technological preparation of manufacturing. The plant that uses computer aid in this production phase is able respond very quickly on the change produced assortment.

Today's industry has brought a new thinking of designers in which simple geometric shaped parts are connected to groups and these are substituted by one complex shaped part. The choice of production technology in this case has a major impact on the costs of production and also on the main period of production.

\section{Technology Selection}

The activities of technologists during the application of decision analysis within the technology selection may vary and they has to be concerned not only on the requirements of the product (design, configuration, quality, accuracy, material, etc.), but they also has to reflect on the plant conditions (represented by the accessible working equipments, employees, etc.). ${ }^{3}$

Minimum cost and maximum productivity of production requires minimizing of the number of used machines, nonstandard jigs and fixtures, tools, etc. As for mass production, first is minimized the number of orientations, maximized the number of simultaneously working tools, the operators with high productivity ratios and automation degree are preferred. So the application of suitable technological method is the possibility to achieve expressive savings in mechanical engineering production.

One of the most exacting technological processes as for energy and material in the mechanical engineering production is the machining. It is not possible to achieve demanded accuracy and machined surface quality at the manufacturing of workpieces by mechanical working or casting process, because the skin is adversely affected. ${ }^{10}$

That's why the machining for giving precision to the sizes is inevitable. There are materials which are not possible to weld or to treat by mechanical working in the mechanical engineering practice. ${ }^{4,9}$ But the material in the mechanical engineering, which is not possible to work by the machining almost doesn't exist, what evidences the versatility of the machining. The versatility and flexibility of the machining is given so that it is possible to work also complex shapes of details by the simple cutting-tool and by the controlled move of the tool or of the workpiece. ${ }^{5,13}$

To be the application of technological strategy maximal flexible from the view of plant request, it is the most suitable to use the $\mathrm{NC}$ machines for mass production of complex part with specific properties of the material. Today designer usually creates the specification for the product as a 3D model. Detailing decides the manufacturing requirements for the product by making a drawing. Next traditional data flow in mechanical engineering can be summarized into these steps: ${ }^{8,12}$

- The information about a product is imported into the CAM system. Usually 3D CAD model is imported. 
- CAM module is used to calculate toolpath for material cutting. CNC programmer just specifies machining operations and the CAM system creates the toolpath, usually written in CL (Cutter Location) data file.

- Calculated toolpath is imported to the postprocessor which converts the CL data to the NC program - the specific machine codes that are required to operate numerically controlled machine tools. Machine codes vary by machine tool.

- NC program written in a notation called G-code is exported to the $\mathrm{NC}$ machine and the manufacturing process can begin.

The postprocessor is one of the most important links in the $\mathrm{CNC}$ machining process. The company can have best programming system, the best DNC system, and the most accurate machines, but without dependable postprocessors the code getting to its machine could cause problems. Most machines require some tweaking to the postprocessor to make it to produce code to user's likings and programming habits. Most CAM software comes with built in postprocessor; however there are many stands alone postprocessor. It is the last software link between an ideal CAD model and a "real" machined part. Unfortunately there are some problems with using of postprocessors, as are e.g.:

- absence of the ready postprocessor in a CAD/CAM system for specific "machine tool / machine control" combination,

- it is very difficult and often impossible to modify the postprocessor available in the CAD/CAM system to the specific "machine tool / machine control" combination. In general, the technologist is forced to permanently correct wrong NC program file with the text processor,

- usually generalized postprocessors of majority of the $\mathrm{CAD} / \mathrm{CAM}$ systems are very difficult to adapt to $\mathrm{NC} / \mathrm{CNC}$ equipment, made in $70-80$ years.

- each new CAD/CAM system requires an individual postprocessor for the same NC equipment, so the company has to pay twice for the same stuff,

- creating a custom postprocessor for each unique machine is often difficult and costly.

One of the manners how to simplify the data flow and data translation in the future is the introducing of STEP NC into production. STEP NC defines a CNC part program as a series of operations that remove material defined by features. The features supported include holes, slots, pockets and volumes defined by 3D surfaces. Each operation contributes to the manufacture of a feature by defining the volume of material to be removed, the tolerances, the type of tool required and some basic characteristics such as whether this is a roughing or finishing operation. The operations are then sequenced into a work plan that converts the stock into the final part. STEP NC allows a complete database of machining information to be built around the digital product model and ultimately makes it possible for this enhanced product model to serve as machine tool input. This database is structured such that part features are linked to specific "working steps," generic descriptions of various machining operations. STEP $\mathrm{NC}$ working steps are roughly equivalent to machining commands formatted as traditional $M$ and $G$ codes. ${ }^{6}$ On the basis of listed above it is visible that for the manufacturing of complex shaped parts by means of CNC machines is useful to create $3 \mathrm{D}$ model serving for CL data generation.

\section{Reverse Engineering}

Engineering is the process of designing, manufacturing, assembling and maintaining products and systems. There are two types of engineering: forward and reverse. Forward engineering is the traditional process of moving from highlevel abstractions and logical designs to the physical implementation of system. While conventional engineering transforms engineering concepts and models into real parts, in reverse engineering real parts are transformed into such computer models which are suitable to exploit all advantages of modern $\mathrm{CAD} / \mathrm{CAM}$ technologies and transfer real objects into virtual reality environment.

In some situations, there may be a physical part (product) without any technical details, such as drawings, bills-ofmaterial or without engineering data. The process of duplicating an existing part, subassembly or product, without drawings, documentation or a computer model is known as Reverse engineering. Reverse engineering is also defined as the process of obtaining a geometric CAD model from 3D points acquired by scanning (digitizing) existing object. ${ }^{1}$

This approach is difficult and time consuming, but it is getting easier all the time thanks to IT, for two reasons:

- Firstly, as engineering techniques themselves become more computerised, more of the design is due to the computer. Thus, recognisable blocks of code, or groups of circuit elements on a substrate, often occur in many different designs produced by the same computer program. These are easier to recognise and interpret than a customised product would be.

- Secondly, artificial intelligence techniques for pattern recognition, and for parsing and interpretation, have advanced to the point where these and other structures within a product can be recognised automatically.

However, whilst it is often possible to automate the generation of a higher level structural description of a product, recognizing what it is doing is difficult and still requires human skills, and it may simply not be possible to recapture some parts of original specification to which the product was made by studying the product. Since reverse engineering still needs human input, at some stage the reverse engineering process needs to produce a complete system description of the product, to allow a human to work out how the product functions; it is only after this human analysis that the product can be split into its component parts.

The digitization of real objects is possible due to scanning equipment which enables the conversion of the real three dimensional objects into a digital form. 3D scanners are the devices that analyze a real-world object or environment to collect data on its shape and possibly its appearance. ${ }^{2}$ Many 
different technologies can be used to build these 3D scanning devices; each technology comes with its own limitations, advantages and costs. It should be remembered that many limitations in the kind of objects that can be digitized are still present: for example optical technologies encounter many difficulties with shiny, mirroring or transparent objects. Contact 3D scanners probe the subject through physical touch. Non-Contact Active scanners emit some kind of radiation or light and detect its reflection in order to probe an object or environment. Possible types of emissions used include light, ultrasound or x-ray. The fastest scanners are the laser ones. Also, a significant factor is the size of the scanned component or, possibly the mobility of the scanning equipment. The majority of scanners are limited by scanning space in which it is possible to scan. The principle of the majority of these equipments is based on the scanning the object's surface in its discrete points and it follows that digitized object is presented on a computer as a large number of points in space (point cloud).

The advantages of real part digitizing can be summarized into the following points: ${ }^{7,14}$

- The optimization of its structural solution before the production, its quick modification.

- The utilisation of the object in the assembly allows detecting the conflicts with other components not only in static, but in kinematics state, too, so in marginal constrains of the motion.

- The defining of the couples, loadings, materials and other $3 \mathrm{D}$ model properties enables to execute the various types of analysis (structural, thermal, dynamic...) on the object and so predicts the object behaviour in real conditions.

- It is possible to simulate the machining process by means of the created 3D models and so to find out the collisions between the tool and the piece.

- As it was said above, one of the major advantages of 3D model is the possibility to generate CL data and consequently to generate the $\mathrm{NC}$ program for the selected control system in very short time.

\section{Problem Definition}

The concept of mass production of complex shaped prototype can be applied on the templates for the stator windings of electromotor for electric household appliances. In Figure 1 is shown front and back view on the two prototypes of the templates.

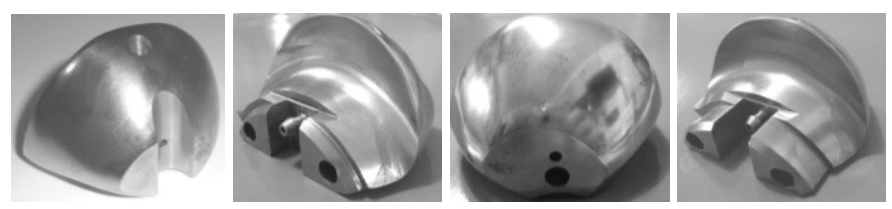

Fig.1. Templates for stator winding of electromotor

Only basic outside dimensions of prototypes and the position of auxiliary features, which are necessary for their arrangement into the assembly, were known. The parts were produced by means of hand grinding into an anti-template and their applicability was tested in real conditions. The process of hand grinding and calibration by means of anti-template repeated several times up to the phase, when the prototype's geometry didn't achieve the needed shape. Consequently two couples of the templates are mirror fixed to the assembly. The magnet wire slides over the top surfaces; the back parts of the templates are the places for wire positioning. The numbers of wire loops define the output of electromotor. Schematic 3D model of assembly in the working position with several loops of wire and also the final real version of motor winding are shown in Figure 2.
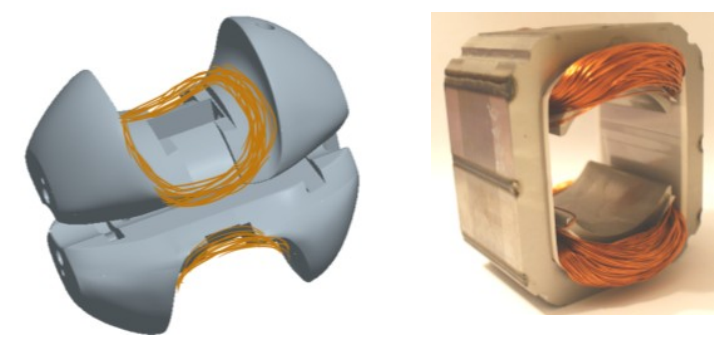

Fig.2. Templates in working position and the final real version of motor winding

The mode of usage of presented parts in real practice requires to make them from high alloyed tool steel with high precision; therefore the casting couldn't be selected as production technology. With regard of available sponsor plant opportunities and its machinery and also in view of the complex form of the parts, it has appeared to select 5-axes milling as the most suitable production technology. Unfortunately the NC program creation was at the beginning not possible (neither in manual way nor from CL data generation) because the dimensions of complex surfaces and their shapes were undefined, respectively undocumented.

\section{Problem Solution}

The unknown geometrical characteristic obtaining at the complex shaped parts is time consuming process. The concept of this process consists of several steps. Some of them are:

1. Real part preparation for scanning

2. Process of scanning

3. Data processing and transferring

4. 3D model creation in selected CAD/CAM system

5. CL data generation

Before scanning process starts it is necessary to solve some problems as are for example:

- reflex and shiny face of real part causes that laser beam reflects back and the surface can't be scanned. In this case it is needed to tarnish the part for example by spraying, not however by black colour, because that laser beam absorbs.

- material, from which the physical model is made, should not be from transparent material (such as for example glass).

- combination of concave and convex surfaces on one part caused that laser beam is not able to recover the shape as 
one whole entity. The solution of this problem is repeated scan of the object in various positions and with various settings. The combination of planar and rotating scan modes provides the possibility to scan already objects with difficult shapes.

- size respectively the weight of scanned object in respect to technical parameters of scanner.

Considering the surface of the original component was too reflexive for the laser beam (as it was polished), it was necessary to decrease its gloss values, e.g. by spray-painting it with a gray undercoat colour. At the same time, it was essential to evenly apply the sprayed layer as this factor may also affects the approximation rate of a created model toward its original and a finite accuracy of the component created on the basis of a virtual 3D model. Scanner software often allows: ${ }^{2}$

- to translate one complex shaped surface through a cloud (a grid) of scanned points, whilst the accuracy of coverage depended on the number of selected checkpoints,

- to modify the polygonal meshes by means of editing control points, polygon edges and surfaces (removing, moving or adding new surfaces),

- to reduce the polygonal meshes, i.e. a reduction of the number of polygons in the meshes, however, at the expense of the quality and display fidelity,

- to fill the cracks which arose at the scanning in polygonal meshes on the basis of the NURBS surface definition, and repair a partially insufficient representation of the scanned data,

- to partially polish the obtained model, however, not with a sufficient accuracy,

- to display the scanned object in four versions: as a point cloud, wire, hide lines and rendering.

However, after the scanning, the virtual radius surfaces and the transition curves were not smooth. (Figure 3)
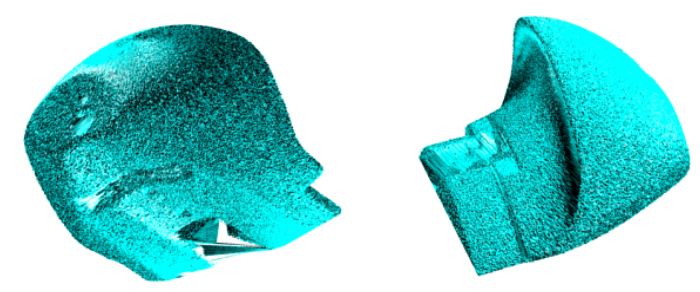

Fig.3. Part after the scanning

Geometric data describing the established surface in 3D environment were neither applicable for various types of analyses, nor for a CL data generation. As a result, it was necessary to import them to a selected CAD/CAM system, in order to their further processing. It is advantageous to use the surface operations for work with complex shaped design within the selected software application. For this purpose, in parallel sections were created the curves that defined the profile of "top surface" on an imported model. Curves of Bezier and Spline types were used most often at work as they best represented the imported template shape. Through a point set and via a mathematical apparatus the interpolating or approximating curves, were translated. (Figure 4)

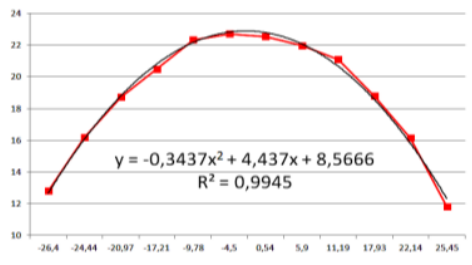

Fig.4. Example of interpolation and the curve definition by polynomial function

The curves were covered with a coat surface which was created as a CAD/CAM system's own element and it was also possible to control, analyse and modify the curvature and "smoothness" of the selected surface. (Figure 5)
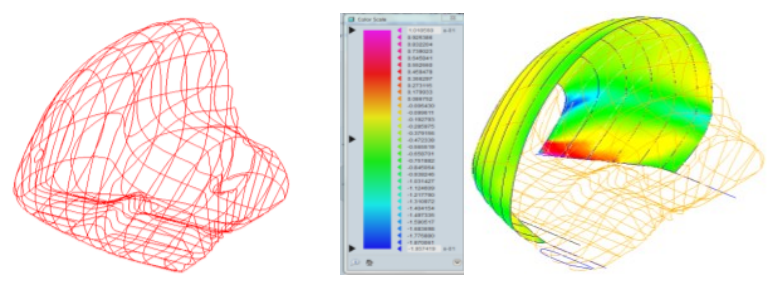

Fig.5. Basic curves for surface creation and its analyzing

The final versions of 3D models are presented in Figure 6.
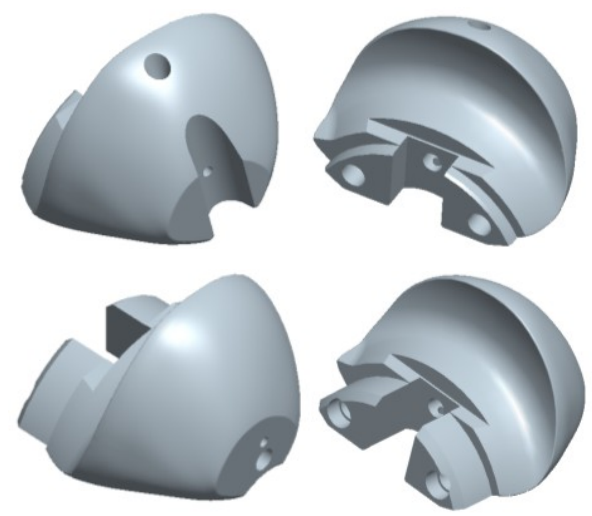

Fig.6. Final shape of the 3D models

The geometry description of virtual model was exported from CAD/CAM system to STL format that was used for the producing of silicone form and consequently new physical part was made by Rapid Prototyping method. (Figure 7)

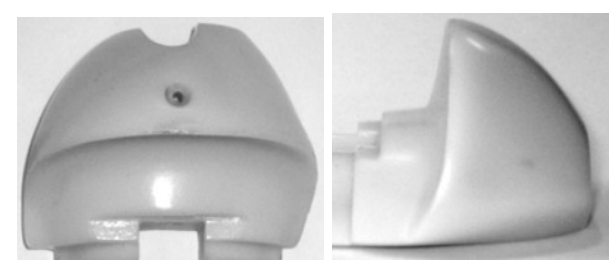

Fig.7. Plastic part produced by Rapid protoyping 
The plastic part was compared with the primary real prototype inside the CAD/CAM system (Figure 8a) and also by means of $3 \mathrm{D}$ measuring equipment (Figure $8 \mathrm{~b}$ ).

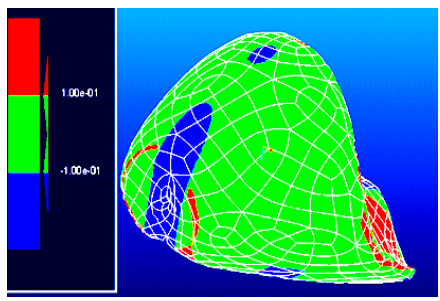

a)

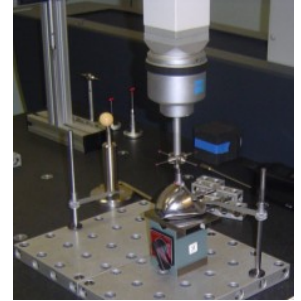

b)
Fig.8. Comparison of 3D model and scanned part

It was possible to allege that the model corresponds to the real steel part in required accuracy. So CL data as a direct output of CAM module were transformed by postprocessor into the readable information for controller of concrete $\mathrm{CNC}$ machine and applied as NC program.

\section{Conclusions}

The automation of the manufacturing is one of the main goals in present days, what is enabled by the quickly development of information technology and by the sequential application of computer aid into all areas of the production. Many of the hand-made prototypes are created without drawing documentation and so the manufacturing of such real parts is very complicated. The utilization of computer techniques can become basis for suggestion of new manufacturing technology, especially if the mass production is necessary. The suitable using of the modern methods, technologies, equipments (computers, NC machines, scanners,...) and its correlative combination can greatly decrease delivery time of parts, investment quantity blocked in stores and costingness to its pandering. ${ }^{11}$ The possibility of costs evaluation and their influence from the view of model utilization within Reverse engineering method is in the Figure 9. ${ }^{1}$

After the creating of 3D model and after the generating of $\mathrm{NC}$ program, the terms of delivery can be shortened about 90 till $98 \%$, the number of storage pieces can be decreased about $50 \%$ and the costs for the production can markedly suffer decline.

It can be said that utilization of this concept was successfully introduced in real conditions of plant and the templates for stator windings of electromotors are produced by means of new method today.

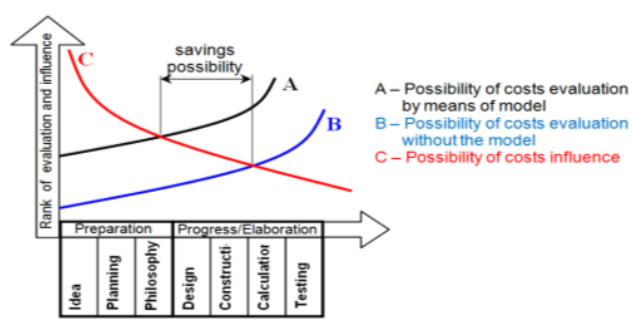

Fig.9. Possibility of costs evaluation and their influence ${ }^{1}$

\section{Acknowledgments}

Paper originates with the direct support of Ministry of Education of Slovak republic by grants KEGA 035TUKE4/2011 KEGA and also it is the result of the Project implementation: Competency Centre for Knowledge technologies applied in Innovation of Production Systems in Industry and Services, ITMS: 26220220155, supported by the Research \& Development Operational Programme funded by the ERDF.

\section{References}

[1] S. Babjak, The planning of reverse engineering in quick product development system I., Transfer of innovation, Vol. 9, p. 59-61 (2006)

[2] M. Dubravcik, The equipment of digitizing, Transfer of innovation, N. 8, p. 52-54 (2005)

[3] M. Belan, A. Tarasovicova, The utilization procedural gaseous medium in machining of steel 1.4301 by tools from HSS, Annals of DAAAM Vienna, Austria, p. 0639-0640 (2011)

[4] D. Jakubeczyova et al., Testing of thin PVD coatings deposited on pm speed steel, Chemical lists, Vol. 105, No. 16, p. 618-620 (2011)

[5] J. Jurko, A. Panda, M. Gajdos, T. Zaborowski, Verification of cutting zone machinability during the turning of a new austenitic stainless steel, Advances in computer science and education application, Vol. 202/2, p. 338-345 (2011)

[6] Kishinami, T. et al., XML-based High Level NC Data Modelling for Manufacturing Data Management, International Conference on Intelligent Autonomous Systems, Italy (2000)

[7] R. Krehel, J. Dobransky, Application of data analysis process in identification system of surface topography, In: Manufacturing Technologies, Vol. 14, p. 116-119 (2010)

[8] J. Mascenik, S. Gaspar, CA technologies as effective tool in production process, Ai Magazine Vol. 4, No. 2, p. 86-87 (2011)

[9] M. Neslusan, I. Mrkvica, R. Cep et al., Deformations after heat treatment and their influence on cutting process, Technical Gazette, Vol. 18, No. 4, p. 601-608 (2011)

[10] A. Paulikova, et al., Quality assessment of cutting fluids with regard to factors of indoor working environment, Journal of Production Engineering, Vol. 12, No.1 (2009)

[11] K. Senderska et al., Module principle of composition of manual assembly stations, Technologia i automatyzacja montažu, No. 1, p. 29-32 (1997)

[12] B. Slowinski, K. Nadolny, Effective Manufacturing Method for Automated Inside Diameter Grinding, Journal of Advanced Mechanical Design, Systems, and Manufacturing, Vol. 1, No. 4, p. $472-480$ (2007)

[13] Z. Somsakova, et al., Machining of Wood Plastic Composite, Materiale plastice, Vol. 49, No. 1, p.55-57 (2012)

[14] J. Valicek, S. Hloch, D. Kozak, Surface geometric parameters proposal for the advanced control of abrasive waterjet technology, International Journal of Advanced Manufacturing Technology, Vol. 41, No. 3-4, p.323-328. (2009) 